\title{
Hadamard's variational formula in terms of stress and strain tensors
}

\author{
Björn Gustafsson ${ }^{1}\left[\right.$ [ $\cdot$ Ahmed Sebbar ${ }^{2,3}$
}

Received: 11 September 2021 / Accepted: 5 December 2021 / Published online: 5 January 2022

(C) The Author(s) 2021

\begin{abstract}
Starting from a Lagrangian action functional for two scalar fields we construct, by variational methods, the Laplacian Green function for a bounded domain and an appropriate stress tensor. By a further variation, imposed by a given vector field, we arrive at an interior version of the Hadamard variational formula, previously considered by P. Garabedian. It gives the variation of the Green function in terms of a pairing between the stress tensor and a strain tensor in the interior of the domain, this contrasting the classical Hadamard formula which is expressed as a pure boundary variation.
\end{abstract}

Keywords Green function · Hadamard formula - Stress tensor · Strain tensor · Energy momentum tensor - Lie derivative

Mathematics Subject Classification 53A45 $\cdot$ 53B10 $\cdot 70$ S05

\section{Introduction}

The Hadamard variational formula expresses how the Green function for a domain changes under an infinitesimal variation of the boundary of the domain. It is usually

Harold S. Shapiro, in memoriam.

In memoriam: We believe that this paper is much in spirit of the interests and work of Harold S. Shapiro. In fact, during his long carrier at KTH, Harold S. Shapiro gave several doctoral courses, and also gave topics for doctoral theses, inspired by that book of P. Garabedian out of which this paper grew.

\footnotetext{
$凶$ Björn Gustafsson

gbjorn@kth.se

Ahmed Sebbar

sebbar@chapman.edu; ahmed.sebbar@math.u-bordeaux.fr

1 Department of Mathematics, KTH, 10044 Stockholm, Sweden

2 Department of Mathematics, Chapman University, Orange, CA 92866, USA

3 IMB, UMR 5251, Université Bordeaux, F-33405 Talence, France
}

Dirkhäuser 


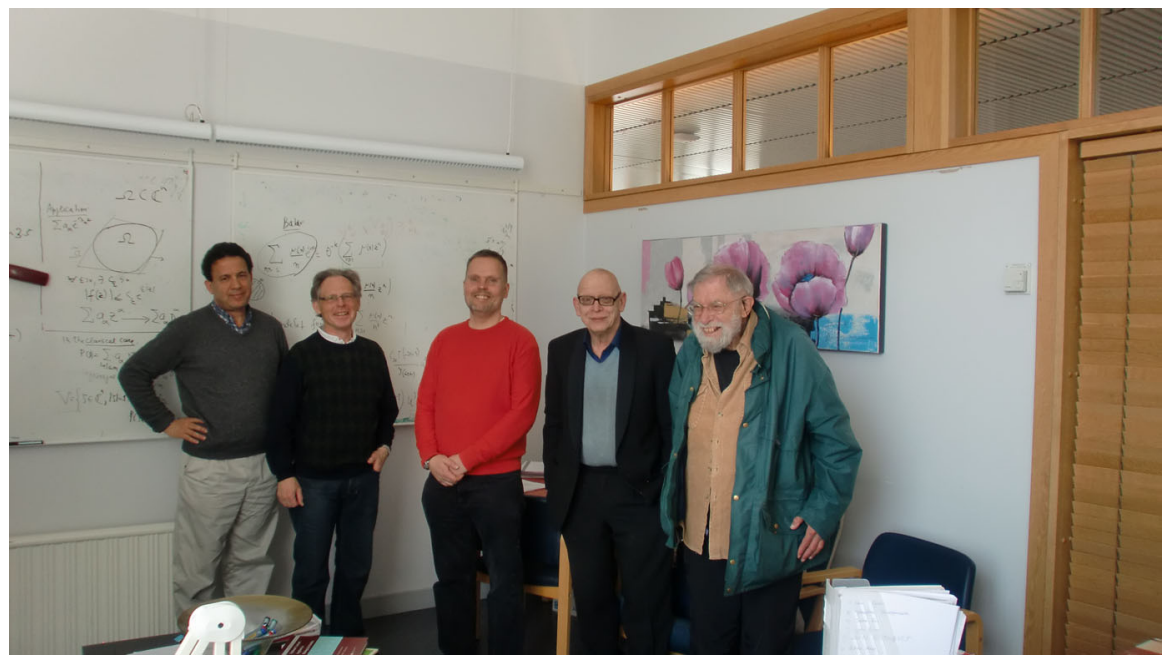

Fig. 1 Harold S. Shapiro with some friends (colleagues and visitors) at the mathematical department of KTH, probably in April 2012. From left: Ahmed Sebbar, Björn Gustafsson, Håkan Hedenmalm, Jan-Erik Björk, Harold S. Shapiro. Photo taken by Henrik Shahgholian (in his office)

formulated in terms of a boundary integral, like in (2.2) below. However, in his book [2], Paul Garabedian formulated the principle instead in terms of an area integral (in two dimensions) containing a generalization of the (Maxwell) stress tensor, a kind of energy-momentum tensor for the electromagnetic field (see [3]). The present paper, written as a tribute to Harold S. Shapiro (Fig. 1), grew out from attempts to understand Garabedian's point of view from a more general perspective.

We elaborate the subject in a setting of subdomains of a Riemannian manifold of arbitrary dimension using tools of differential geometry and tensor analysis. The main result of the paper, Theorem 6.1, expresses the Hadamard principle in terms of a bulk integral containing a stress tensor and a strain tensor. The Green function takes the role of being a physical scalar field (or potential), and it is the main ingredient in a Lagrangian action functional representing a polarized energy. In addition, the Green function turns out to coincide (except for a sign) with the value of the action at extremum. The stress tensor is obtained by varying the action with respect to the underlying Riemannian metric, while the strain tensor represents the information of how an imposed vector field deforms the domain.

\section{Hadamard formula in Euclidean setting}

The (Laplacian) Green function $G_{a}$ for a (bounded) domain $\Omega \subset \mathbb{R}^{n}$ is defined by the properties

$$
\begin{aligned}
-\Delta G_{a}=\delta_{a} & \text { in } \Omega, \\
G_{a}=0 & \text { on } \partial \Omega .
\end{aligned}
$$


Writing $G(x, a)=G_{a}(x), G(x, a)$ is symmetric with respect to $x$ and $a$. This is most clearly seen by using standard Green's formulas to express the Green function as a mutual energy:

$$
G(a, b)=\int_{\Omega}\left(\nabla G_{a} \cdot \nabla G_{b}\right) d x .
$$

The Green function certainly depends on the domain, $G=G_{\Omega}$, and Hadamard's classical formula [2] expresses how $G_{\Omega}(x, a)$ changes under small variations of the boundary of this domain. In traditional notations it reads

$$
\delta G(a, b)=\int_{\partial \Omega} \frac{\partial G(\cdot, a)}{\partial n} \frac{\partial G(\cdot, b)}{\partial n} \delta n d \sigma,
$$

where $\delta n$ represents an infinitesimal deformation of $\partial \Omega$ in the outward normal direction. To express this more accurately one may divide by an infinitesimal time interval $\delta t$ so that $v_{n}=\delta n / \delta t$ represents the velocity of the boundary in normal direction under an evolution $\Omega(t)$ with respect to $t$. Then the formula becomes

$$
\frac{d}{d t} G_{\Omega(t)}(a, b)=\int_{\partial \Omega} \frac{\partial G(\cdot, a)}{\partial n} \frac{\partial G(\cdot, b)}{\partial n} v_{n} d \sigma .
$$

For the derivation of (2.3) it is useful to think of $v_{n}$ as the normal component of a vector field $\mathbf{v}$ which is defined everywhere, and then let all of $\Omega \cup \partial \Omega$ move with $\mathbf{v}$. In particular the boundary points move, and since we shall not keep track of individual points on the boundary the effective meaning simply becomes that the speed of the boundary $\partial \Omega$ in the normal direction equals the normal component $v_{n}=\mathbf{v} \cdot \mathbf{n}$ of $\mathbf{v}$ on $\partial \Omega$.

Thus the tangential component of $\mathbf{v}$ on $\partial \Omega$ is insignificant for (2.3). The same is true for the interior points of $\Omega$ : the restriction of $\mathbf{v}$ to $\Omega$ never enters the formula. This is exactly what marks the difference between the formula (2.3) and the formula given in [2]. The latter formula is based on the strain on the points in $\Omega$ caused by $\mathbf{v}$. This strain makes up a strain tensor $D_{i j}$, and together with a certain stress tensor $T^{i j}$ the variational formula becomes

$$
\frac{d}{d t} G_{\Omega(t)}(a, b)=\int_{\Omega} T^{i j} D_{i j} d x+\text { source terms. }
$$

See more precisely Theorem 6.1 below. Garabedian's formula appears as equation (15.20) in [2], and the stress tensor there is also given in our Example 3.1.

\section{Several variations of an action functional}

We shall put the variational formula (2.2) in a context of field theory, where we vary a Lagrangian action functional with respect to all fields involved. The action is

$$
S=\int_{\Omega} \nabla \psi_{a} \cdot \nabla \psi_{b}-\psi_{a}(b)-\psi_{b}(a),
$$


a polarized energy for two real-valued scalar fields and provided with source terms (point sources at $a$ and $b$ ). The fields are to vanish on the boundary:

$$
\psi_{a}=\psi_{b}=0 \text { on } \partial \Omega
$$

Variation of $S$ with respect to $\psi_{a}, \psi_{b}$ and requiring it to be stationary to the first order (i.e. setting $\delta S=0$ in traditional notation) gives

$$
-\Delta \psi_{a}=\delta_{a}, \quad-\Delta \psi_{b}=\delta_{b},
$$

hence that $\psi_{a}, \psi_{b}$ are actually the Green functions at $a$ and $b$ :

$$
\psi_{a}=G_{a}=G(\cdot, a), \quad \psi_{b}=G_{b}=G(\cdot, b) .
$$

From this, together with (2.1), we see that "on-shell" (i.e. with (3.3) inserted) the action equals the Green function itself, modulo a sign:

$$
S=G(a, b)-G(b, a)-G(a, b)=-G(a, b) .
$$

This is a negative number, which is natural since setting $\delta S=0$ should mean that the action is minimized. Notice that $\psi_{a}=\psi_{b}=0$ are allowed test functions.

In relativistic field theory one often introduces energy-momentum tensors by varying an action with respect to the underlying Minkowskian metric. In our case there is no time variable present, and it is more appropriate to speak of just a stress tensor (or possibly stress-energy tensor), and this can then be introduced on an abstract basis by varying the underlying Riemannian metric. So far we have not seen any metric, but the Euclidean metric $d s^{2}=d x_{1}^{2}+\cdots+d x_{n}^{2}$ actually is there, implicit in the scalar product and the nabla operator. When varying this Euclidean metric we get more general Riemannian metrics. Therefore it is natural that we, from outset, let $\Omega$ be a subdomain of a Riemannian manifold $M$.

We shall need notations from differential geometry, in particular those of tensor analysis and differential forms. We shall then write coordinates with upper indices, like $x^{1}, \ldots, x^{n}$, and we write the metric as

$$
d s^{2}=g_{i j}(x) d x^{i} \otimes d x^{j},
$$

with $\left(g_{i j}\right)$ symmetric and positive definite at each point. Summation over repeated indices (when one is up, the other down) is implied.

In this setting the action functional (3.1) becomes

$$
S=\int_{\Omega} \frac{\partial \psi_{a}}{\partial x^{i}} \frac{\partial \psi_{b}}{\partial x^{j}} g^{i j} \sqrt{g} d x-\psi_{a}(b)-\psi_{b}(a) .
$$


In (3.5) we have also used the metric tensor with upper indices. By definition, $\left(g^{i j}\right)$ represents the inverse of $\left(g_{i j}\right)$ when these tensors are viewed as a matrices:

$$
g_{i j} g^{j k}=g_{i}^{k}= \begin{cases}1 & (i=k) \\ 0 & (i \neq k)\end{cases}
$$

Moreover, $g=\operatorname{det}\left(g_{i j}\right)$ denotes the determinant of $\left(g_{i j}\right)$, and

$$
d x=d x^{1} \wedge \cdots \wedge d x^{n}
$$

This $n$-form depends on the choice of coordinates, while there is an invariant version, namely the volume form given by

$$
\operatorname{vol}^{n}=\sqrt{g} d x
$$

The manifold is assumed to be oriented, and of course we choose $\sqrt{g}>0$.

If we want to spell out all dependencies for $S$ we may write

$$
S=S\left[\psi_{a}, \psi_{b} ;\left(g_{i j}\right) ; \Omega\right]
$$

We have already varied $S$ with respect to $\psi_{a}$ and $\psi_{b}$, this was elementary in the Euclidean setting and it extends directly to the Riemannian case. See (4.2) and thereafter for some details and coordinate expressions.

The variation with respect to $\left(g_{i j}\right)$ is also standard, but for the sake of completeness we shall give the details. In fact, this is what makes the stress tensor pop up. When varying $S$ with respect to $\left(g_{i j}\right)$ it is convenient to think of $\left(g_{i j}\right)$ as depending on a real parameter, say $t$, and write $g_{i j}=g_{i j}(t)$. We shall only make variations which keep $\left(g_{i j}\right)$ symmetric. Thus, on denoting $t$-derivatives by a dot whenever convenient we have $\dot{g}_{i j}=\dot{g}_{j i}$.

Now $\frac{d}{d t}\left(g_{i j} g^{j k}\right)=0$, hence $\dot{g}_{i j} g^{j k}+g_{i j} \dot{g}^{j k}=0$. Therefore,

$$
\dot{g}^{k \ell}=-g^{k i} \dot{g}_{i j} g^{j \ell}, \quad \dot{g}_{i j}=-g_{i k} \dot{g}^{k \ell} g_{\ell j}
$$

In general, if $A$ is an $n \times n$ matrix, assumed here to be symmetric with positive eigenvalues (just for simplicity), then

$$
\begin{aligned}
\frac{d}{d t}(\operatorname{det} A) & =\frac{d}{d t}\left(e^{\log \operatorname{det} A}\right)=e^{\log \operatorname{det} A} \cdot \frac{d}{d t}(\log \operatorname{det} A) \\
& =\operatorname{det} A \cdot \frac{d}{d t}(\operatorname{tr} \log A)=\operatorname{det} A \cdot \operatorname{tr} \frac{d}{d t}(\log A)=\operatorname{det} A \cdot \operatorname{tr}\left(A^{-1} \dot{A}\right)
\end{aligned}
$$

With $A=\left(g_{i j}\right), g=\operatorname{det}\left(g_{i j}\right)$ this gives

$$
\dot{g}=g g^{i j} \dot{g}_{i j}
$$


It also follows that

$$
\frac{d}{d t} \sqrt{g}=\frac{\dot{g}}{2 \sqrt{g}}=\frac{1}{2} \sqrt{g} g^{i j} \dot{g}_{i j} .
$$

Starting from (3.5) and using (3.8), (3.9) we now have

$$
\begin{aligned}
& \frac{d}{d t} S\left[\psi_{a}, \psi_{b} ;\left(g_{i j}(t)\right) ; \Omega\right]=\frac{d}{d t} \int_{\Omega} \frac{\partial \psi_{a}}{\partial x^{k}} \frac{\partial \psi_{b}}{\partial x^{\ell}} g^{k \ell} \sqrt{g} d x \\
& \quad=\int_{\Omega}\left(-\frac{\partial \psi_{a}}{\partial x^{k}} \frac{\partial \psi_{b}}{\partial x^{\ell}} g^{k i} \dot{g}_{i j} g^{j \ell}\right) \sqrt{g} d x+\frac{1}{2} \int_{\Omega} \frac{\partial \psi_{a}}{\partial x^{k}} \frac{\partial \psi_{b}}{\partial x^{\ell}} g^{k \ell} \sqrt{g} g^{i j} \dot{g}_{i j} d x \\
& \quad=\frac{1}{2} \int_{\Omega}\left(-\frac{\partial \psi_{a}}{\partial x^{k}} \frac{\partial \psi_{b}}{\partial x^{\ell}} g^{k i} g^{j \ell}-\frac{\partial \psi_{a}}{\partial x^{k}} \frac{\partial \psi_{b}}{\partial x^{\ell}} g^{k j} g^{i \ell}+\frac{\partial \psi_{a}}{\partial x^{k}} \frac{\partial \psi_{b}}{\partial x^{\ell}} g^{k \ell} g^{i j}\right) \dot{g}_{i j} \sqrt{g} d x \\
& \quad=-\frac{1}{2} \int_{\Omega} T^{i j} \dot{g}_{i j} \sqrt{g} d x .
\end{aligned}
$$

Here we have defined the stress tensor in contravariant form (upper indices) as that tensor $T^{i j} \frac{\partial}{\partial x^{i}} \otimes \frac{\partial}{\partial x^{j}}$ which has components

$$
T^{i j}=\frac{\partial \psi_{a}}{\partial x^{k}} \frac{\partial \psi_{b}}{\partial x^{\ell}} g^{k i} g^{j \ell}+\frac{\partial \psi_{a}}{\partial x^{k}} \frac{\partial \psi_{b}}{\partial x^{\ell}} g^{k j} g^{i \ell}-\frac{\partial \psi_{a}}{\partial x^{k}} \frac{\partial \psi_{b}}{\partial x^{\ell}} g^{k \ell} g^{i j} .
$$

In covariant form (lowered indices) it is the tensor $T_{i j} d x^{i} \otimes d x^{j}$ with

$$
T_{i j}=\frac{\partial \psi_{a}}{\partial x^{i}} \frac{\partial \psi_{b}}{\partial x^{j}}+\frac{\partial \psi_{a}}{\partial x^{j}} \frac{\partial \psi_{b}}{\partial x^{i}}-\frac{\partial \psi_{a}}{\partial x^{k}} \frac{\partial \psi_{b}}{\partial x^{\ell}} g^{k \ell} g_{i j}
$$

Example 3.1 When $n=2$ and $g_{i j}=\delta_{i j}$ we get, on setting $\psi_{a}=G_{a}, \psi_{b}=G_{b}$, $x=x^{1}, y=x^{2}$,

$$
\left(\begin{array}{ll}
T_{11} & T_{12} \\
T_{21} & T_{22}
\end{array}\right)=\left(\begin{array}{l}
\frac{\partial G_{a}}{\partial x} \frac{\partial G_{b}}{\partial x}-\frac{\partial G_{a}}{\partial y} \frac{\partial G_{b}}{\partial y} \frac{\partial G_{a}}{\partial x} \frac{\partial G_{b}}{\partial y}+\frac{\partial G_{a}}{\partial y} \frac{\partial G_{b}}{\partial x} \\
\frac{\partial G_{a}}{\partial x} \frac{\partial G_{b}}{\partial y}+\frac{\partial G_{a}}{\partial y} \frac{\partial G_{b}}{\partial x} \frac{\partial G_{a}}{\partial y} \frac{\partial G_{b}}{\partial y}-\frac{\partial G_{a}}{\partial x} \frac{\partial G_{b}}{\partial x}
\end{array}\right)
$$

This is exactly the expression given in Garabedian [2].

\section{Classical Hadamard by Lie derivatives}

The final step now is to vary the action (3.5), (3.7) with respect to the domain $\Omega$, and so obtain the Hadamard formula. This step becomes more elegant when expressed in the language of differential forms.

We let the smoothly bounded domain $\Omega=\Omega(t) \subset M$ move in the flow of a vector field $\mathbf{v}=\sum_{j=1}^{n} v^{j} \frac{\partial}{\partial x^{j}}$, and denote by $\mathcal{L}_{\mathbf{v}}$ the Lie derivative, and by $i(\mathbf{v})$ interior derivation ("contraction"), with respect to v. See in general Frankel [1] for differential geometric concepts and notations. 
In terms of differential forms the representation (2.1) of the Green function becomes

$$
G(a, b)=\int_{\Omega} d G(\cdot, a) \wedge * d G(\cdot, b),
$$

the star being the Hodge star operator. When acting on a one-form $v=v_{j} d x^{j}$, the Hodge star is related to interior derivation with the corresponding vector field $\mathbf{v}=v^{k} \frac{\partial}{\partial x^{k}}$ by $* v=i(\mathbf{v}) \operatorname{vol}^{n}$. This makes the $n$-form

$$
d * v=\left(\sqrt{g} v^{k}\right)_{, k} d x=v_{; k}^{k} \sqrt{g} d x=(\operatorname{div} \mathbf{v}) \operatorname{vol}^{n}
$$

have the role of being the "divergence" of $\nu$. Above we have used some standard tensor analysis notations, like

$$
v_{, j}^{k}=\frac{\partial v^{k}}{\partial x^{j}}, \quad v_{; j}^{k}=v_{, j}^{k}+\Gamma_{j \ell}^{k} v^{\ell}
$$

for ordinary and covariant derivatives (respectively), with

$$
\Gamma_{j \ell}^{k}=\frac{1}{2} g^{k i}\left(g_{\ell i, j}+g_{i j, \ell}-g_{j \ell, i}\right)
$$

denoting the "Christoffel symbols" (connection coefficients). Implicit in (4.2) is the crucial identity $\left(v^{k} \sqrt{g}\right)_{, k}=v_{; k}^{k} \sqrt{g}$. For the Laplacian of a function $\phi$ we have, similarly,

$$
d * d \phi=\frac{\partial}{\partial x^{k}}\left(\sqrt{g} g^{k j} \frac{\partial \phi}{\partial x^{j}}\right) d x=\phi_{; k j} g^{k j} \sqrt{g} d x=\Delta \phi \operatorname{vol}^{n} .
$$

We remark also that $\operatorname{vol}^{n}=* 1$.

Now the action functional becomes

$$
S=\int_{\Omega} d \psi_{a} \wedge * d \psi_{b}-\psi_{a}(b)-\psi_{b}(a)
$$

Here the Riemannian metric is not visible, but it is built into the Hodge operator. Varying $S$ in (4.3) with respect to $\psi_{a}$ (for example) gives, by partial integration, that

$$
-d * d \psi_{b}=\varepsilon_{b},
$$

where $\varepsilon_{b}$ denotes the Dirac measure at $b$ regarded as a $n$-form current. The relation to $\delta_{b}$ as a Dirac "function" (or distribution) is

$$
\varepsilon_{b}=\delta_{b} \operatorname{vol}^{n}=\delta_{b} \sqrt{g} d x
$$

In view of (3.4) it is a matter of taste whether one performs the variation with respect to $\Omega$ in the equation (4.1) for $G(a, b)$ or in the expression (4.3) for $S$, but it 
is slightly more elegant and general to work directly with (4.3) as far as possible. To simplify notation we set

$$
\alpha=\alpha_{j} d x^{j}=d \psi_{a}, \quad \beta=\beta_{j} d x^{j}=d \psi_{b}, \quad \Phi=\alpha_{k} \beta^{k}
$$

Thus $\alpha_{j}=\partial \psi_{a} / \partial x^{j}, \beta_{j}=\partial \psi_{b} / \partial x^{j}$. Clearly $d \alpha=d \beta=0$ in $\Omega$, and $\psi_{a}, \psi_{b}$ being constant (zero) on the boundary give that $\alpha=\beta=0$ along $\partial \Omega$.

Let

$$
d \sigma=\operatorname{vol}^{n-1}=i(\mathbf{n}) \operatorname{vol}^{n}
$$

denote the surface area element on $\partial \Omega$ when this is regarded as a manifold in itself, and where $\mathbf{n}$ denotes the outward unit normal vector on $\partial \Omega$. We then interprete

$$
\begin{aligned}
& i(\mathbf{v}) \alpha=\frac{\partial \psi_{a}}{\partial n} v_{n} \quad \text { on } \partial \Omega, \\
& * \beta=\frac{\partial \psi_{b}}{\partial n} d \sigma \quad \text { along } \partial \Omega .
\end{aligned}
$$

Two basic properties of the Lie derivative are the "homotopy" formula

$$
\mathcal{L}_{\mathbf{v}}=d \circ i(\mathbf{v})+i(\mathbf{v}) \circ d
$$

(when acting on differential forms) and the fact that for a domain $\Omega(t)$ (or chain of integration of any sort) moving in the flow of $\mathbf{v}$,

$$
\frac{d}{d t} \int_{\Omega(t)}(\ldots)=\int_{\Omega(t)} \mathcal{L}_{\mathbf{v}}(\ldots)
$$

In the notations (4.4) the action $S$ takes the form

$$
S=\int_{\Omega} \alpha \wedge * \beta-\psi_{a}(b)-\psi_{b}(a)=\int_{\Omega} \Phi \operatorname{vol}^{n}-\psi_{a}(b)-\psi_{b}(a) .
$$

From this it follows that

$$
\begin{aligned}
\frac{d S}{d t} & =\int_{\Omega} \mathcal{L}_{\mathbf{v}}(\alpha \wedge * \beta)=\int_{\Omega}(d \circ i(\mathbf{v})+i(\mathbf{v}) \circ d)(\alpha \wedge * \beta) \\
& =\int_{\Omega} d(i(\mathbf{v})(\alpha \wedge * \beta))+0=\int_{\partial \Omega} i(\mathbf{v})(\alpha \wedge * \beta) \\
& =\int_{\partial \Omega}(i(\mathbf{v}) \alpha) \wedge * \beta-\int_{\partial \Omega} \alpha \wedge i(\mathbf{v})(\beta)=\int_{\partial \Omega} \frac{\partial \psi_{a}}{\partial n} \frac{\partial \psi_{b}}{\partial n} v_{n} d \sigma-0
\end{aligned}
$$

This result can be rewritten as

$$
\frac{d}{d t} \int_{\Omega(t)} d \psi_{a} \wedge * d \psi_{b}=\int_{\partial \Omega} \frac{\partial \psi_{a}}{\partial n} \frac{\partial \psi_{b}}{\partial n} v_{n} d \sigma
$$


We never used that $\psi_{a}, \psi_{b}$ eventually are to be the Green functions of $\Omega$, but inserting finally (3.3) and using (4.1) gives the Hadamard formula in its classical form (2.3).

\section{Divergence of stress tensor}

In terms of $\alpha, \beta, \Phi$ in (4.4) the covariant version (3.11) of the stress tensor can be written

$$
T_{i j}=\alpha_{i} \beta_{j}+\alpha_{j} \beta_{i}-g_{i j} \alpha_{k} \beta^{k}=\alpha_{i} \beta_{j}+\alpha_{j} \beta_{i}-\Phi g_{i j}
$$

The trace of $T$ is

$$
\operatorname{tr} T=T_{i j} g^{i j}=(2-n) \Phi
$$

and the Green function becomes, with (3.3) in force,

$$
G(a, b)=\int_{\Omega} \alpha \wedge * \beta=\int_{\Omega} \Phi \operatorname{vol}^{n} .
$$

The contravariant version (3.10) of the stress tensor has components

$$
T^{i j}=T_{r s} g^{r i} g^{s j}=\alpha^{i} \beta^{j}+\alpha^{j} \beta^{i}-\Phi g^{i j}
$$

The divergence of this tensor is obtained by contracting the second index with the covariant derivative:

$$
\operatorname{div} T=T_{; j}^{i j} \frac{\partial}{\partial x^{i}} .
$$

Using the fact that all covariant derivatives of the metric tensor vanish and that, by (4.4), $\alpha_{i ; j}=\alpha_{j ; i}, \beta_{i ; j}=\beta_{j ; i}$ we have

$$
T_{; j}^{i j}=\alpha_{; j}^{i} \beta^{j}+\alpha^{i} \beta_{; j}^{j}+\alpha_{; j}^{j} \beta^{i}+\alpha^{j} \beta_{; j}^{i}-\Phi_{; j} g^{i j}=\alpha^{i} \beta_{; j}^{j}+\beta^{i} \alpha_{; j}^{j}
$$

When $\psi_{a}=G_{a}, \psi_{b}=G_{b}$, so that $\alpha_{; j}^{j}=\Delta \psi_{a}=-\delta_{a}, \beta_{; j}^{j}=\Delta \psi_{b}=-\delta_{b}$, equation (5.5) becomes

$$
T_{; j}^{i j}=-\alpha^{i} \delta_{b}-\beta^{i} \delta_{a}
$$

The right member in (5.6) can be interpreted as a source concentrated at the points $a$ and $b$. More precisely, it is a vector field with distributional coefficients (a vector current) composed by the isolated vector $-\nabla G_{a}$ sitting (like a point charge) at the point $b$ and the vector $-\nabla G_{b}$ sitting at $a$. In summary we have

Lemma 5.1 When $\psi_{a}=G_{a}, \psi_{b}=G_{b}$ the divergence of the stress tensor vanishes except for the two point source field given in (5.6). Expressed in vector notation:

$$
\operatorname{div} T=-\left(\nabla G_{a}\right) \delta_{b}-\left(\nabla G_{b}\right) \delta_{a}
$$




\section{Hadamard in terms of stress and strain tensors}

In the final computation in Sect. 4 we had an integral over $\Omega$ involving a Lie derivative, and this integral was pushed to the boundary. But there is also the possibility not to go to the boundary. Then also the vector field $\mathbf{v}$ becomes differentiated, and one may arrange matters so that the derivatives of $\mathbf{v}$ appear only in a certain strain tensor $D$. This is to be paired with the stress tensor $T$ discussed in Sects. 3 and 5 .

The stress and strain tensors are the main actors in linear elasticity theory, where the basic result, Hooke's law (first formulated in 1678), expresses that the stress and strain tensors are proportional (more exactly, linearly related) to each other for an elastic material (see in general [4]).

Given a vector field $\mathbf{v}$, thought of as representing an infinitesimal deformation of some material, the corresponding strain tensor $D$ is the symmetric covariant tensor defined by

$$
2 D=2 D_{i j}(x) d x^{i} \otimes d x^{j}=\mathcal{L}_{\mathbf{v}}\left(g_{i j} d x^{i} \otimes d x^{j}\right) .
$$

The components of $D$ are given by

$$
2 D_{i j}=g_{i k} v_{; j}^{k}+g_{k j} v_{; i}^{k}=v_{i ; j}+v_{j ; i} .
$$

Using this we can now formulate the following generalization of equation (15.20) in [2].

Theorem 6.1 The variation of the Green function $G_{\Omega}(a, b)$ due to a deformation of $\Omega \subset M$ driven by a smooth vector field $\mathbf{v}$ is, in terms of the stress tensor $T=T(a, b)$ and the strain tensor $D=D(\mathbf{v})$, given by

$$
\frac{d}{d t} G_{\Omega(t)}(a, b)=\int_{\Omega} T^{i j} D_{i j} \operatorname{vol}^{n}-\mathbf{v}\left(G_{a}\right)(b)-\mathbf{v}\left(G_{b}\right)(a)
$$

In the right member $\mathbf{v}=v^{j} \frac{\partial}{\partial x^{j}}$ is regarded as a derivation (directional derivative).

Proof Using (5.3) and (4.2) (essentially) we first have

$$
\begin{aligned}
\frac{d}{d t} G_{\Omega(t)}(a, b) & =\int_{\Omega} \mathcal{L}_{\mathbf{v}}(\alpha \wedge * \beta)=\int_{\Omega} \mathcal{L}_{\mathbf{v}}\left(\Phi \operatorname{vol}^{n}\right) \\
& =\int_{\Omega} d\left(i(\mathbf{v}) \Phi \operatorname{vol}^{n}\right)=\int_{\Omega} d\left(i(\Phi \mathbf{v}) \operatorname{vol}^{n}\right)=\int_{\Omega}\left(\Phi v^{j}\right)_{; j} \operatorname{vol}^{n}(6.3)
\end{aligned}
$$

The next step is to show that

$$
\int_{\Omega}\left(\Phi v^{j}\right)_{; j} \operatorname{vol}^{n}=\int_{\Omega}\left(T^{i j} v_{i}\right)_{; j} \operatorname{vol}^{n}
$$

This will be achieved by pushing the difference between the two members to the boundary, after which cancellations will make it disappear. 
Let $\mathbf{n}$ denote the outward unit normal vector on $\partial \Omega$ and let $n_{j} d x^{j}$ be the corresponding one-form. It may be realized as $n_{j} d x^{j}=d u$ for a function $u$ which is defined near $\partial \Omega$, vanishes on $\partial \Omega$, and increases away from $\Omega$ with $|\nabla u|=1$ on $\partial \Omega$. (One may take $u=-G_{a} /\left|\nabla G_{a}\right|$, for example.) Then inserting (4.4), (5.4) and using Stokes' formula we have

$$
\begin{aligned}
& \int_{\Omega}\left(T^{i j} v_{i}\right)_{; j} \operatorname{vol}^{n}-\int_{\Omega}\left(\Phi v^{j}\right)_{; j} \operatorname{vol}^{n} \\
& =\int_{\Omega}\left(\left(\alpha^{i} \beta^{j}+\alpha^{j} \beta^{i}-\alpha_{k} \beta^{k} g^{i j}-\alpha^{k} \beta_{k} g^{i j}\right) v_{i}\right)_{; j} \operatorname{vol}^{n} \\
& =\int_{\Omega}\left(\left(\alpha^{i} \beta^{j}-\alpha_{k} \beta^{k} g^{i j}\right) v_{i}+\left(\alpha^{j} \beta^{i}-\alpha^{k} \beta_{k} g^{i j}\right) v_{i}\right)_{; j} \operatorname{vol}^{n} \\
& =\int_{\partial \Omega}\left(\left(\alpha^{i} \beta^{j}-\alpha_{k} \beta^{k} g^{i j}\right) v_{i}+\left(\alpha^{j} \beta^{i}-\alpha^{k} \beta_{k} g^{i j}\right) v_{i}\right) n_{j} d \sigma \\
& =\int_{\partial \Omega} \beta^{j}\left(\alpha_{i} n_{j}-\alpha_{j} n_{i}\right) v^{i} d \sigma+\int_{\partial \Omega} \alpha^{j}\left(\beta_{i} n_{j}-\beta_{j} n_{i}\right) v^{i} d \sigma=0 .
\end{aligned}
$$

In the last step we used that, along the boundary $\partial \Omega$,

$$
\alpha_{i} d x^{i}=\beta_{i} d x^{i}=n_{i} d x^{i}=0,
$$

hence that the covectors with components $\alpha_{i}, \beta_{i}, n_{i}$ are proportional at each point of $\partial \Omega$. From this it follows that

$$
\alpha_{i} n_{j}=\alpha_{j} n_{i}, \quad \beta_{i} n_{j}=\beta_{j} n_{i} \text { for all } i, j .
$$

Thus (6.4) is now established.

Finally, using the symmetries of $T$ and $D$ together with (5.6), (5.7) we can continue the right member of (6.4) by

$$
\begin{aligned}
\int_{\Omega}\left(T^{i j} v_{i}\right)_{; j} \mathrm{vol}^{n} & =\int_{\Omega}\left(T_{; j}^{i j} v_{i}+T^{i j} v_{i ; j}\right) \mathrm{vol}^{n} \\
& =\int_{\Omega}\left(-\alpha^{i} \delta_{b}-\beta^{i} \delta_{a}\right) v_{i} \mathrm{vol}^{n}+\int_{\Omega} T^{i j} D_{i j} \mathrm{vol}^{n} \\
& =-\mathbf{v}\left(G_{a}\right)(b)-\mathbf{v}\left(G_{b}\right)(a)+\int_{\Omega} T^{i j} D_{i j} \mathrm{vol}^{n}
\end{aligned}
$$

Combing this with (6.3), (6.4) completes the proof.

Acknowledgements The first author expresses warm thanks for a generous invitation to Chapman University, where this work was initiated, and for the creative and friendly atmosphere which he experienced during the stay.

Funding Open access funding provided by Royal Institute of Technology.

\section{Declaration}

Data availability statement All data needed are contained in the manuscript. 
Conflict of interest: All authors certify that they have no affiliations with or involvement in any organization or entity with any financial interest or non-financial interest in the subject matter discussed in this manuscript.

Open Access This article is licensed under a Creative Commons Attribution 4.0 International License, which permits use, sharing, adaptation, distribution and reproduction in any medium or format, as long as you give appropriate credit to the original author(s) and the source, provide a link to the Creative Commons licence, and indicate if changes were made. The images or other third party material in this article are included in the article's Creative Commons licence, unless indicated otherwise in a credit line to the material. If material is not included in the article's Creative Commons licence and your intended use is not permitted by statutory regulation or exceeds the permitted use, you will need to obtain permission directly from the copyright holder. To view a copy of this licence, visit http://creativecommons.org/licenses/by/4.0/.

\section{References}

1. Frankel, T.: .The Geometry of Physics, Cambridge University Press, Cambridge, third ed., . An introduction, (2012)

2. Garabedian, P.R.: Partial differential equations. John Wiley \& Sons Inc, New York-London-Sydney (1964)

3. Landau, L. D., Lifshitz, E. M.:, The classical theory of fields, Revised second edition. Course of Theoretical Physics, Vol. 2. Translated from the Russian by Morton Hamermesh, Pergamon Press, Oxford-London-Paris-Frankfurt; Addison-Wesley Publishing Co., Inc., Reading, Mass., (1962)

4. Landau, L.D., Lifshitz, E.M., Course of theoretical physics. Vol. 7, Pergamon Press, Oxford, third, (eds.): Theory of elasticity. Translated from the Russian by J. B. Sykes and W. H, Reid (1986)

Publisher's Note Springer Nature remains neutral with regard to jurisdictional claims in published maps and institutional affiliations. 